\title{
Interaction between an aerodynamically driven, wall-bound drop and a single groove
}

\author{
Patrick M. Seiler, Ilia V. Roisman ${ }^{\mathrm{a}}$, and Cameron Tropea \\ Institute of Fluid Mechanics and Aerodynamics, Technische Universität Darmstadt, \\ Darmstadt, Germany
}

Received 29 November 2019 / Accepted 6 July 2020

Published online 14 September 2020

\begin{abstract}
The interaction between an air-driven, wall-bound drop and a groove in the wall of a channel flow has been investigated experimentally using a high-speed video system. Three major outcomes of drop interaction with the groove are observed: (i) the drop passes over the groove, (ii) the drop is immediately fully captured in the groove or (iii) the drop is captured after first wetting the rear side of the groove. The mechanisms leading to these different outcomes are governed by the aerodynamic drag force, by inertial and gravity forces, and by the adhesion force associated with the substrate wettability. A threshold condition for drop capture is developed, based on the ratio of the typical time for drop passage over the groove to the time for the drop to be sucked into the groove. It has been shown that the probability for drop capture increases for higher Bond numbers.
\end{abstract}

\section{Introduction}

Drop propagation over surfaces with geometric discontinuities such as grooves or roughness elements is important in many practical applications. Examples include air-frame icing [1,2], exterior vehicle water management [3], or the widely known lotus effect $[4,5]$. Although there exists some controversy regarding the terminology when classifying and describing surface morphology [6], in the present study the size of the surface structures is the most relevant distinguishing parameter and furthermore, only simple groove geometries are considered. The groove dimensions are of the same order as the drop diameter; hence the groove can be considered a macroscopic structure.

The main subject of the present study is the propagation of an aerodynamically driven drop over a groove in an otherwise smooth surface. The shear driven propagation of drops has been investigated previously by various researchers [7-9]. In the work of [10] the velocity of a wall-bound drop placed in a turbulent channel flow was derived in the form

$$
\mathrm{Ca}=\mathcal{K}\left(\tilde{u}_{\text {att }}^{2}-\tilde{u}_{0}^{2}\right)^{3 / 2}
$$

a e-mail: roisman@sla.tu-darmstadt.de 
where $\tilde{u}_{\text {att }}$ is a dimensionless attack velocity of the air flow (on a distance from the wall comparable with the drop height), $\tilde{u}_{0}$ is a constant associated with the critical air velocity for drop motion inception, and $\mathcal{K}$ is an empirical constant. The air attack velocity is scaled using the balance of the aerodynamic and capillary forces

$$
\tilde{u}_{\mathrm{att}}=u_{\mathrm{att}}\left[\frac{\varrho_{g} A_{0}}{\sigma d_{0}}\right]^{1 / 2},
$$

where $A_{0}$ and $d_{0}$ are the projected drop area exposed to the air flow, and drop diameter, respectively.

The capillary number $\mathrm{Ca}$ is defined as

$$
\mathrm{Ca}=v \frac{\mu}{\sigma},
$$

where $v$ is the drop propagation velocity. This is one of the main parameters governing the flows influenced by the propagating contact lines. The validity of the scaling expressed by equation (1) is confirmed by very good agreement with the experimental data [10] for a wide range of the experimental conditions.

As for the interaction between such a drop and a groove, recently two studies $[11,12]$ have numerically simulated this situation using a level-set volume of fluid approach, whereas the present study examines the problem experimentally and analytically.

In the present case the groove is rectangular with constant depth, but varying width in the flow direction. The groove is placed in a fully developed turbulent channel flow, in which single drops can be deposited on the lower channel wall and the flow speed can be exactly controlled, determining the aerodynamic stresses acting on the drop. The aim of the study is to experimentally determine under what conditions the propagating drop will be captured by the groove, or portions of the liquid will pass over the groove. A model is then presented to predict 'capture' or 'pass', establishing the correct scaling parameters for describing the phenomenon.

\section{Experimental setup and procedure}

\subsection{Experimental setup}

The experimental set-up, shown in Figure 1, consists of four components: a channel flow (I), a test section (II), an imaging system (III) and a data acquisition and control (DAC) unit, all of which have been previously described in a study examining the propagation of wall-bound drops over surfaces [10]. The wind tunnel is an open return, blower type, driven by a radial fan. A standard nozzle is placed at the fan intake, providing an accurate measurement of volume flow rate; hence, of the mean flow velocity in the channel test section. The channel test section, with dimensions $\mathcal{W} \times \mathcal{H}=200 \mathrm{~mm} \times 15 \mathrm{~mm}$ and length $0.5 \mathrm{~m}$, is preceded by a $1.2 \mathrm{~m}$ long channel to ensure that the turbulent channel flow is fully developed in the mean. The lower floor of the test section allows different surfaces to be mounted, for instance to vary the contact angle.

This setup allows for channel Reynolds numbers (based on the test section height $\mathcal{H}$ ) between $3000<\operatorname{Re}_{\mathcal{H}}<32,000$. The normalized velocity profile at the centerline of the channel for $\operatorname{Re}_{\mathcal{H}}=20,000$ is shown in Figure 2. The velocity profile is determined using the hot wire technique for various Reynolds numbers of the flow. The normalized profiles almost coincide for different Reynolds numbers and also agree very well with 


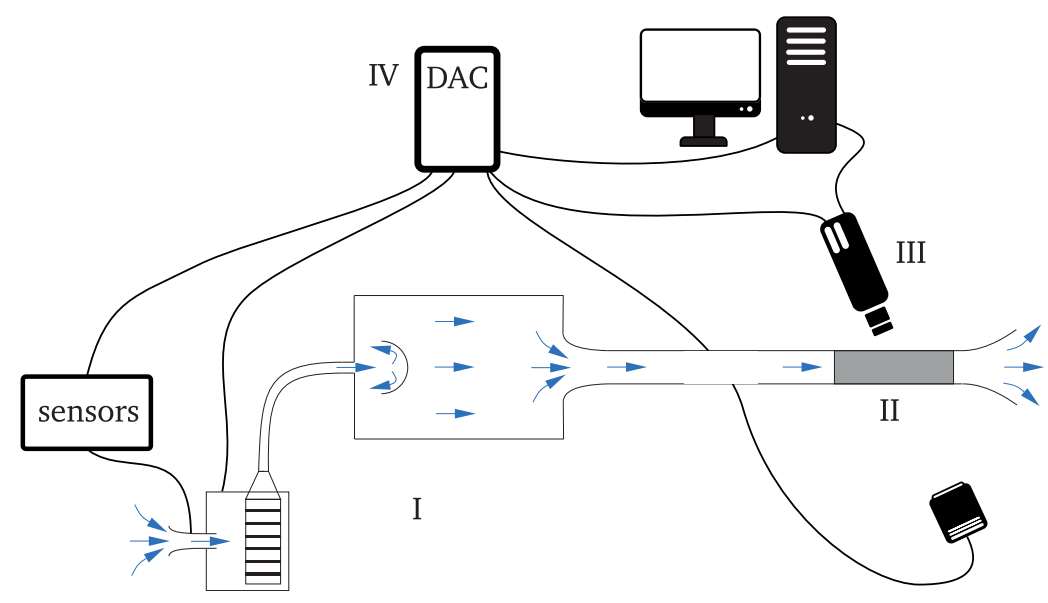

Fig. 1. Experimental setup consisting of the channel flow I, test section II, observation system III and data acquisition and control (DAC) system IV.

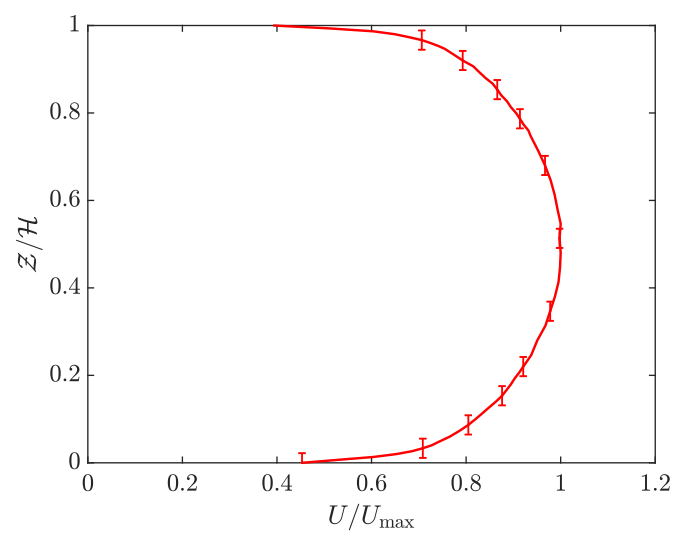

Fig. 2. Normalized hot-wire measurements of the velocity profile in the test section for $\operatorname{Re}_{\mathcal{H}}=20,000$.

the results of the DNS computations [13]. The measurement techniques does not allow to determine the velocity in the close proximity to the wall. The distance to the wall from the first relevant measurement point is $500 \mu \mathrm{m}$. The profile at the lower distances to the wall is then estimated by the interpolation.

The width of the channel $\mathcal{W}=200 \mathrm{~mm}$ is much larger than the typical size of the drops $(\sim 1 \mathrm{~mm})$, used in the experiments. Since the drops are placed at the middle of the channel the corner effects of the flow can be neglected.

The surface used in this study was made of aluminium with an advancing contact angle of $\Theta_{\mathrm{adv}, \mathrm{cr}}=90^{\circ}$ and a receding contact angle of $\Theta_{\text {rec,cr }}=33^{\circ}$. These angles were determined at standard atmospherica conditions by slowly inflating and deflating a single drop using a Krüss DSA 100 measurement system.

To construct the groove two plates were used, one of which was fixed and the other which could be traversed in the flow direction, forming a groove of variable width. The groove width $\delta$ is set by a micrometer caliper and the depth remained constant at $5 \mathrm{~mm}$. A sketch of the groove adjustment is shown in Figure 3. The contact angle of the groove walls is $\Theta_{\mathcal{G}}=70^{\circ}$. It differs from the surface angles because the walls of the groove were machined during manufacturing. 


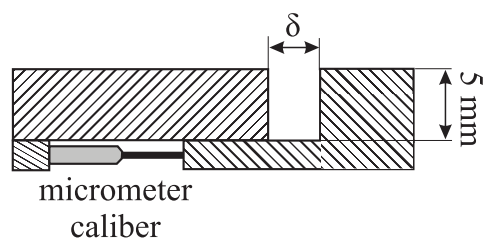

Fig. 3. Sketch of the variable groove.

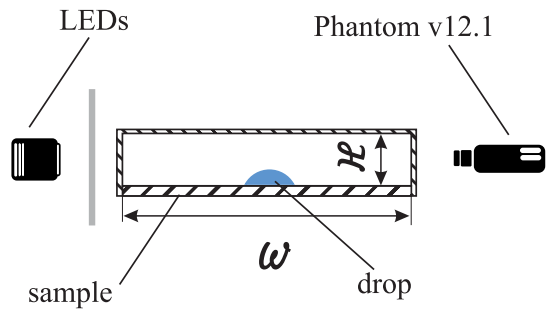

Fig. 4. Sketch of the test section cross section with the camera configuration used in the experiments.

The test section is optically accessible from both walls as well as from above, allowing shadowgraphy observation of the liquid drop. A Phantom v12.1 high speed camera and an LED array was used to capture drop movement at 1000 frames per second (fps). This configuration is schematically shown in Figure 4. The field of view of the Phantom v12.1 camera extends $25 \mathrm{~mm}$ upstream and $10 \mathrm{~mm}$ downstream of the groove; hence, both the velocity of the drop approaching the groove and the outcome after traversing the groove could be determined from the videos.

Drops were inserted into the channel $150 \mathrm{~mm}$ upstream of the groove using an Eppendorf Research plus pipette and at zero flow velocity. Then the flow velocity was increased to a constant value. The aerodynamic forces acting on the drop resulted in drop motion, attaining a constant velocity before encountering the groove.

In order to measure this constant drop velocity the drop contour was found using a sub-pixel edge detection algorithm [14]. Its velocity was defined by the change over time in position of the mid-point of between the advancing and receding contact lines. Due to the importance of both three-phase contact points they were determined by an algorithm similar to the one suggested by [15].

Further details about the experimental facility and measurement equipment can be found in [13].

\section{Observations of drop groove interactions}

A sequence of shadowgraphy images of a drop passing a groove are shown in Figure 5 . At $t=0 \mathrm{~s}$ the drop approaches the groove with constant velocity. At $t=45 \mathrm{~ms}$ the advancing contact line of the drop pins at the upstream edge of the groove. The aerodynamic force then deforms the drop over the groove $(t=60 \mathrm{~ms})$ while the contact line is pinned at the upstream edge. Next, a new advancing contact line forms on the downstream side, as seen at $t=75 \mathrm{~ms}$. At this point a liquid bridge between both edges has formed. The drop continues its forward motion while a fraction of the drop volume remains in the liquid bridge.

The second type of outcome, a drop being immediately captured by the groove, occurs when the substrate on the downstream side is not wetted. In this case, the 

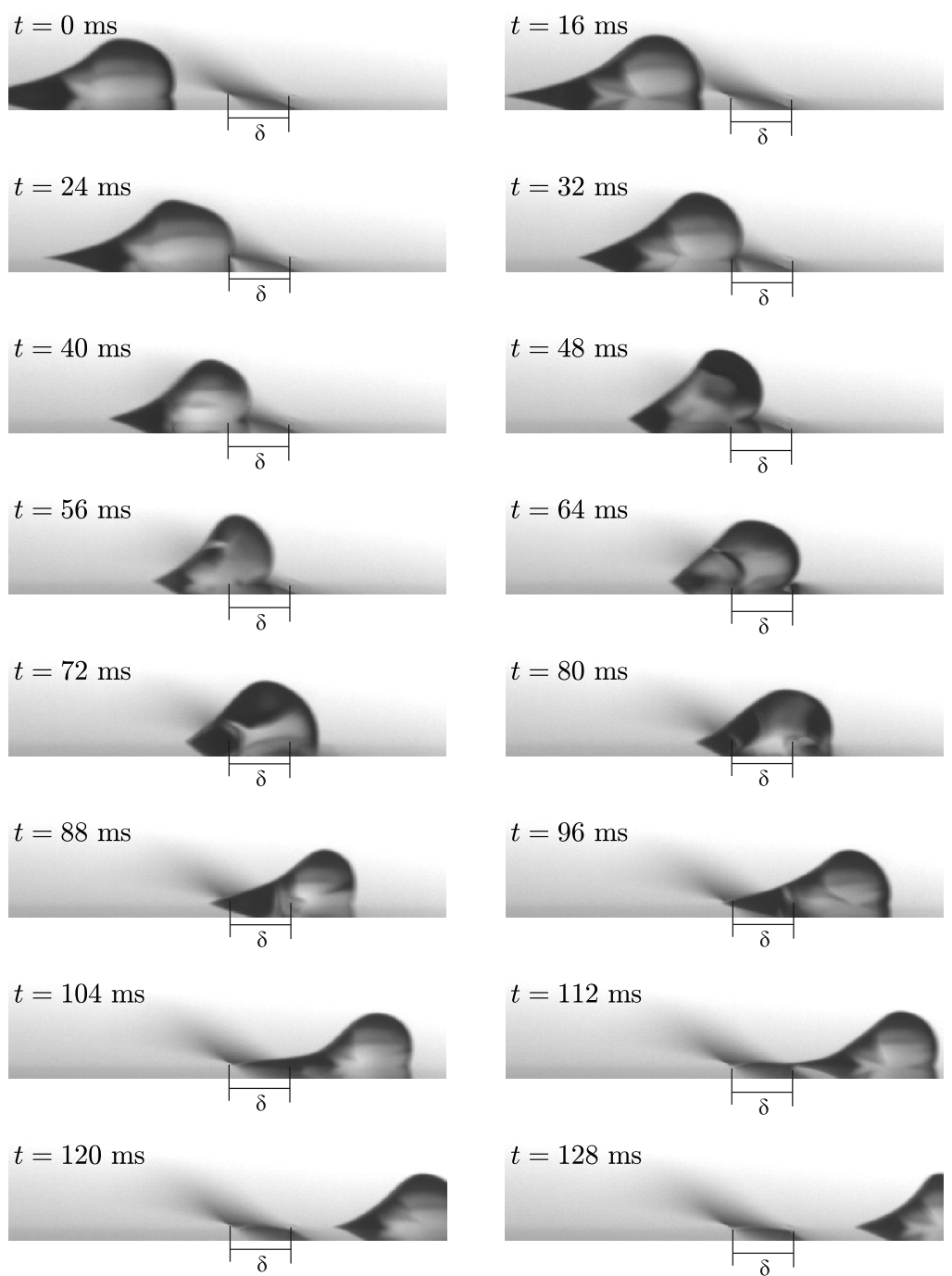

Fig. 5. Series of shadowgraphy images showing a drop groove interaction experiment with a $V_{0}=15 \mu \mathrm{l}$ water drop. The groove has a width of $\delta=1.5 \mathrm{~mm}$. The dimensionless attack velocity is $\tilde{u}_{\text {att }}=1.63$, and the capillary number is $\mathrm{Ca}=1.9 \times 10^{-3}$.

combination of groove width $(\delta)$, drop volume $\left(V_{0}\right)$, and drop velocity $(v)$ either leads to the drop exclusively pinned on the upstream wall of the groove or wetting of the downstream wall while being captured.

A sequence of images showing the third outcome is shown in Figure 6. Similar to the case where the drop passes the groove, a liquid bridge between both sides of the groove is formed. At $t=60 \mathrm{~ms}$ the majority of volume is located over the groove while the substrate on the downstream edge has already been wetted. The capillary forces, dominating in this case, suck the drop into the groove. Since the wetted downstream distance is comparable to the groove width, as seen at $t=9 \mathrm{~ms}$, the force does not suffice to move the contact line on the downstream side. While the drop is sucked into the groove a small droplet remains on the downstream side of the groove. Such minor residuals may remain on both sides of the groove if the dewetting of the substrate can not keep up with the absorption rate of the groove. 

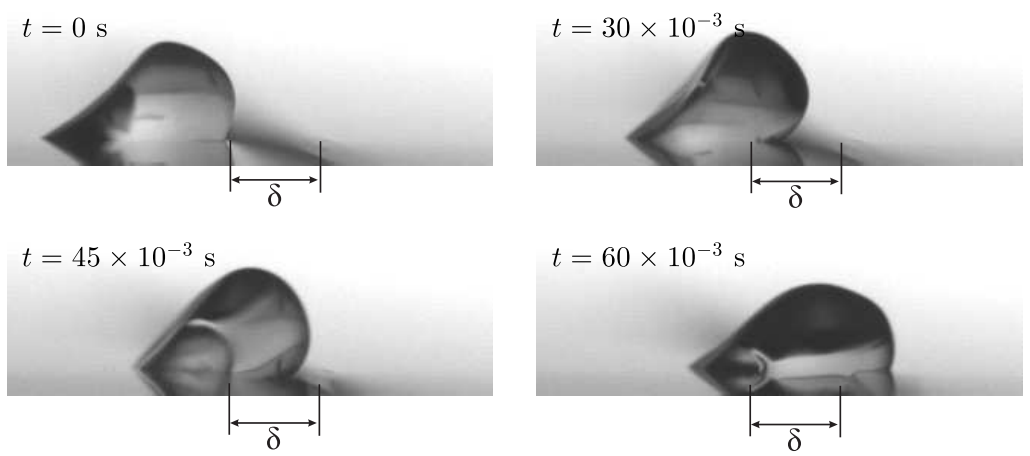

$t=75 \times 10^{-3} \mathrm{~s}$

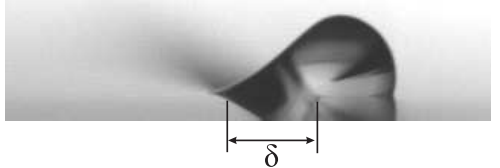

$t=90 \times 10^{-3} \mathrm{~s}$

$t=105 \times 10^{-3} \mathrm{~s}$

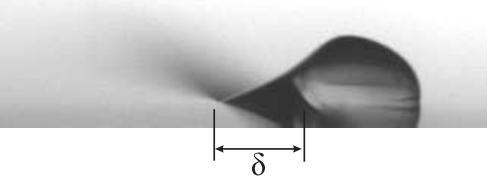

$t=120 \times 10^{-3} \mathrm{~s}$
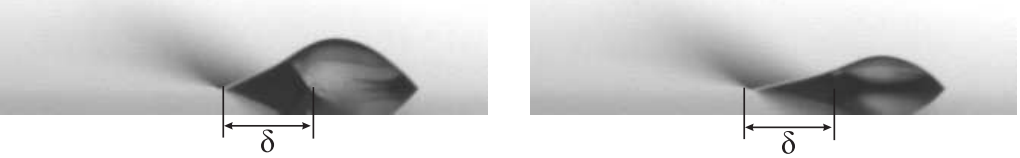

$t=135 \times 10^{-3} \mathrm{~s}$

$t=150 \times 10^{-3} \mathrm{~s}$
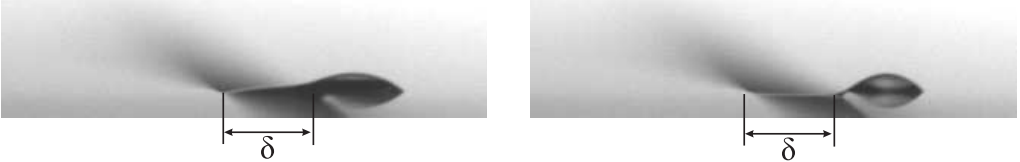

$t=165 \times 10^{-3} \mathrm{~s}$

$t=180 \times 10^{-3} \mathrm{~s}$
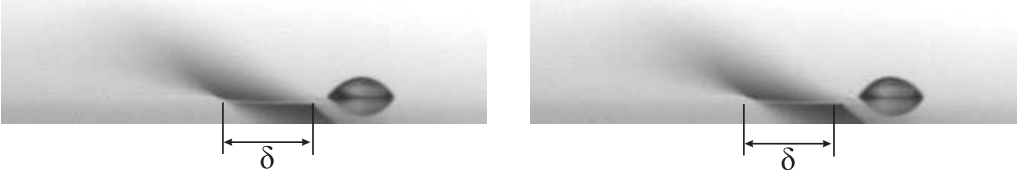

Fig. 6. Series of shadowgraphy images showing a drop-groove interaction experiment with a $V_{0}=15 \mu \mathrm{l}$ water drop. The groove has a width of $\delta=1.5 \mathrm{~mm}$. The dimensionless attack velocity is $\tilde{u}_{\text {att }}=1.57$, and the capillary number is $\mathrm{Ca}=1.7 \times 10^{-3}$.

The visualisations revealed three possible outcomes: only a portion of the liquid enters the groove and the rest continues propagation downstream of the groove; the drop is immediately captured by the groove; or the drop wets the downstream edge of the groove before being captured by it. Sketches of these three outcomes are shown in Figure 7.

\section{Results and discussions}

\subsection{Interaction of a stationary drop with a groove}

When a drop interacts with a groove, part of the drop volume can be sucked into the groove by the forces associated with the groove wettability. The residual drop 
a)

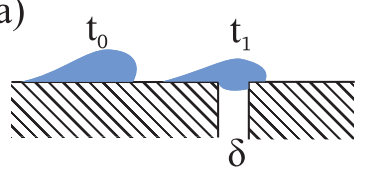

b)

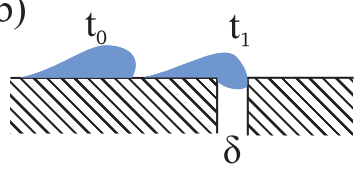

c)

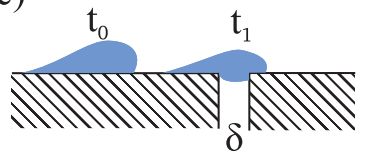

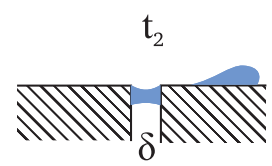

$\mathrm{t}_{2}$

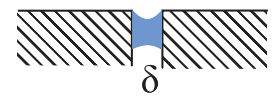

$\mathrm{t}_{2}$

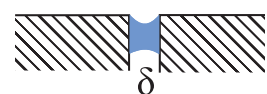

Fig. 7. Sketch of the three outcomes a drop exhibits when encountering a groove: a) drop passes the groove b) drop is immediately captured, c) drop wets the downstream edge and is captured.

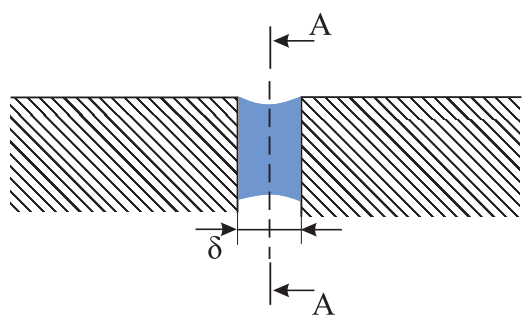

A (cross-section)

Fig. 8. Sketch of the expanding disc during drop absorption.

downstream of the groove can be pinned at the rear corner of the groove. The drop will continue to move further if the residual volume is large enough such that the aerodynamic forces applied to this residual volume is larger than the adhesion forces associated with pinning at the groove edge.

In order to better understand the mechanism of drop suction into the groove a set of experiments was performed on the suction of an initially stationary drop, i.e. without a flow. In the experiments a drop produced by a pipette has been gently placed centrally on the groove. The time for complete suction has been measured for various drop volumes $\left(V_{0}=5 ; 10 ; 15 ; 20 \mu \mathrm{l}\right)$ and groove thicknesses $(\delta=0.1 ; 0.25 ; 0.5 \mathrm{~mm})$.

For these experiments the groove was set up outside of the channel flow and a drop was placed on the groove using a Eppendorf research plus pipette. A video of the absorption was recorded using a Nikon D90 and a Zeiss $50 \mathrm{~mm}$ lens. From the recording, the time $t_{\mathcal{G}}$ required for the drop to be fully absorbed has been determined.

In order to estimate the suction time theoretically, the liquid bridge in the groove is approximated by an expanding disc of radius $R_{\mathcal{G}}(t)$. This approximation is based on the fact that the effect of gravity on the flow in the groove is small, and therefore all directions of flow are equal. A sketch of the expanding disc within the groove is shown in Figure 8.

The average radial velocity of the flow in the groove is $u_{\mathcal{G}}=\dot{R}_{\mathcal{G}} R_{\mathcal{G}} / r$, which satisfies the mass balance equation. Since the Reynolds number of the flow in the groove is much smaller than unity, the inertial terms can be neglected and the pressure 
gradient can be estimated using the lubrication approximation $\partial p / \partial r \approx-12 \mu u_{\mathcal{G}} / \delta^{2}$ [16-18], based on the kinematically admissible parabolic velocity field in the gap.

Then the expression for the pressure in the liquid bridge can be obtained in the form

$$
p_{\mathcal{G}}=p_{0}-\frac{12 \mu R_{\mathcal{G}} \dot{R}_{\mathcal{G}}}{\delta^{2}} \ln \left(\frac{r}{r_{0}}\right)
$$

where $r_{0}$ is the initial radius and $p_{0}$ is the initial pressure at the boundary between the bridge and the drop. Values $r_{0}$ and $p_{0}$ must be introduced to avoid the singularity in the equations at $r=0$.

At large distances from the drop, $r \gg r_{0}$, the value of the logarithmic term in equation (4) varies only weakly. Therefore, as a zero approximation, this term can be expressed by a constant. For large distances from the drop therefore

$$
p_{\mathcal{G}} \approx-\frac{K \mu R_{\mathcal{G}} \dot{R}_{\mathcal{G}}}{\delta^{2}}
$$

where $K$ is a constant.

The pressure at the disk edge is estimated by the wettability of the groove and its thickness. The estimation, with the help of the Young-Laplace equation, yields $p_{\mathcal{G}}\left(r=R_{\mathcal{G}}\right)=-2 \sigma \cos \Theta_{\mathcal{G}} / \delta$. Substitution of this boundary condition into equation (5) yields the following ordinary differential equation for $R_{\mathcal{G}}(t)$

$$
R_{\mathcal{G}}(t) \dot{R}_{\mathcal{G}}=\frac{2 \delta \sigma \cos \Theta_{\mathcal{G}}}{K \mu}
$$

the solution of which is

$$
R_{\mathcal{G}}=\left[\frac{2 \delta \sigma \cos \Theta_{\mathcal{G}}}{K \mu} t\right]^{1 / 2}
$$

Expression (7) allows estimation of the time $t_{\mathcal{G}}$ required for complete capture of the liquid volume $V_{0}$. The radius of the bridge at this instant can be estimated from the mass balance of the drop $V_{0} \approx \pi R_{\mathcal{G}}^{2} \delta / 2$. Expression (7) then yields

$$
t_{\mathcal{G}} \sim \frac{\mu V_{0}}{\delta^{2} \sigma}
$$

The coefficient of proportionality in the linear relation (8), $K / \pi \cos \theta_{\mathcal{G}}$, is of order $10^{3}$.

In Figure 9 the values for the capture time $t_{\mathcal{G}}$ for a static drop are plotted as a function of the term $\mu V_{0} / \delta^{2} \sigma$, determined in equation (8). The linear dependence of these values serves as an experimental confirmation of the scaling expressed by equation (8). The best fit of the experimental data yields for this particular wall wettability

$$
t_{\mathcal{G}} \approx 1036.0 \frac{\mu V_{0}}{\delta^{2} \sigma}
$$




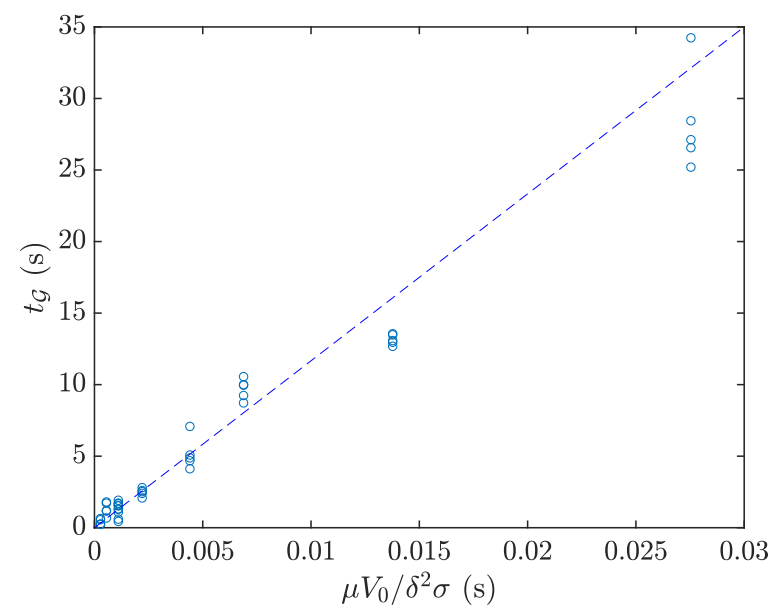

Fig. 9. The measured suction time for a static drop, $t_{\mathcal{G}}$, as a function of the scale $\mu V_{0} / \delta^{2} \sigma$, defined in equation (8).

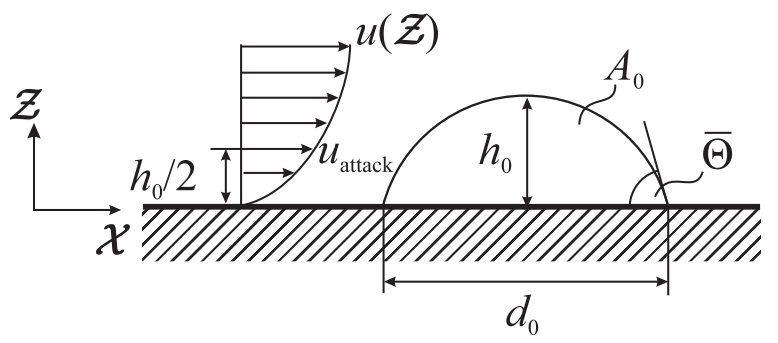

Fig. 10. Sketch of the drop with relevant geometric parameters and attack velocity at half drop height.

\subsection{Interaction of an air-driven drop with a groove}

The characteristic geometric parameters of a drop, its height $h_{0}$, diameter of crosssectional contact area $d_{0}$ and cross-sectional area $A_{0}$, defined in Figure 10, can be roughly estimated for a given drop volume $V_{0}$ and mean contact angle $\bar{\Theta}=\left(\Theta_{\text {adv }, c r}+\right.$ $\left.\Theta_{\text {rec,cr }}\right) / 2$ by approximating its shape as a truncated sphere [10]

$$
\begin{aligned}
& h_{0}=\left[\frac{6 V_{0} \sin ^{2} \frac{\bar{\Theta}}{2}}{\pi(2+\cos \bar{\Theta})}\right]^{1 / 3}, \quad d_{0}=2 h_{0} \cot \frac{\bar{\Theta}}{2}, \\
& A_{0}=h_{0}^{2} \frac{\bar{\Theta}-\sin \bar{\Theta} \cos \bar{\Theta}}{(1-\cos \bar{\Theta})^{2}} .
\end{aligned}
$$

These length scales are correct only for drops with very small Bond numbers which move with a very small velocity, corresponding to small capillary numbers. These are conditions under which the drop will not be significantly deformed. However, these expressions can also be used for the formulation of relevant dimensionless parameters for the description of the hydrodynamic phenomena at higher drop velocities, higher drop deformations and higher Bond numbers.

One of the important parameters influencing the outcome of the interaction with a groove of a moving drop is the ratio of the typical time of the drop passing over the 

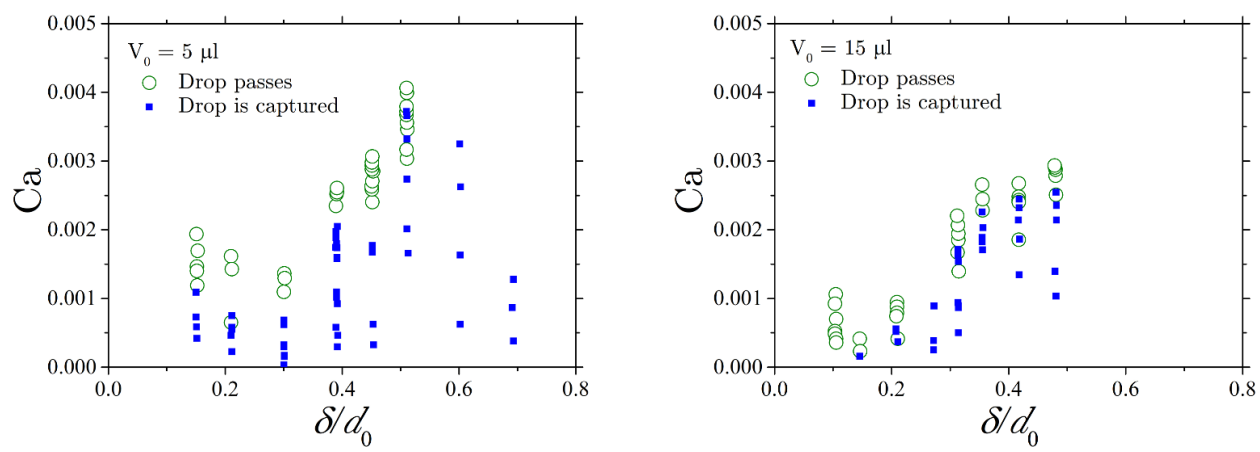

Fig. 11. Examples of the different outcomes of interactions of the drops of the volumes 5 and $15 \mu \mathrm{l}$ with the grooves of different thicknesses.

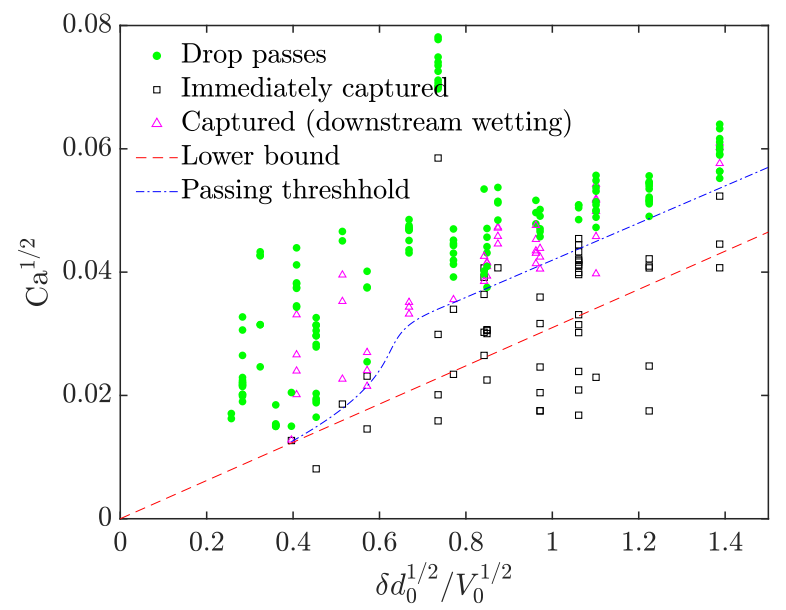

Fig. 12. A nomogram of the major outcomes of the drop interaction with the groove for different capillary numbers, drop and groove sizes, presented in dimensionless form. The lower bound curve to the theoretical estimation (13). Dash-dotted line represents the empirically determined boundary for a drop to pass a groove.

groove, $t_{\mathrm{drop}} \sim d_{0} / v$ and the typical time $t_{\mathcal{G}}$ required for the suction of the entire drop volume. It is obvious that drop passing is not possible if $t_{\mathcal{G}}<t_{\text {drop }}$. The lower bound for the drop passing is determined from the comparison of these two characteristic times with the help of equation (8) as

$$
\frac{t_{\mathcal{G}}}{t_{\mathrm{drop}}}=\frac{1036 \cdot \mu V_{0} v}{\delta^{2} \sigma d_{0}}=1
$$

This condition can be rewritten in the dimensionless form using the definition (3) of the capillary number

$$
\mathrm{Ca}^{1 / 2} \approx 0.031 \frac{\delta d_{0}^{1 / 2}}{V_{0}^{1 / 2}} .
$$

Two nomograms for the outcome of drop interaction with the grooves of different thicknesses are shown in Figure 11 for drop volumes 5 and $15 \mu \mathrm{l}$. In Figure 12 
various outcomes of the interactions of a single drop with a groove are shown for various capillary numbers, drop volumes and groove thicknesses. The outcomes are grouped into three major regimes: drop passes the groove, is immediately captured or captured after downstream wetting. On the nomogram in Figure 12 also a straight line corresponding to the predicted lower bound for drop passing, determined from equation (13), is plotted. It is remarkable that the predicted lower bound is indeed rather close to the threshold conditions for drop passing. Some underestimation can be explained by drop deformation by the aerodynamic forces. Therefore, the drop length is in fact larger than $d_{0}$, estimated from the static drop situation. This increased drop length extends the residence time of the drop over the groove. Since the suction time is proportional to the volume, not drop length, a higher Capillary number would be required in order for the drop to pass the groove.

The scaling shown in Figure 12 reveals the limits for the conditions corresponding to immediate capture. However, it this scaling does not unequivocally distinguish the regimes of drop passing and drop capture, once the rear side of the groove has been wetted. This suggests that the physics of drop capture involves further forces.

Some drops have been captured by the groove even though their velocity exceeded the threshold. Such cases are marked by the magenta triangles in Figure 12. They correspond to drops being captured after the rear side of the groove was wetted, as shown in the example given in Figure 7c. For the same set of the experimental parameters different outcomes, passing or capturing, are observed with a certain probability. Nevertheless, the experiments show that these outcomes are influenced also by gravity and the inertial terms of the liquid in the drop.

The ratio of the inertial terms in the drop and the capillary pressure associated with the groove width is related by the Weber number defined as

$$
\mathrm{We}=\frac{\varrho v^{2} \delta}{\sigma} .
$$

At higher Weber numbers the pressure over the groove entrance is lower due to the fast drop motion and reduced suction speed. Moreover, one can imagine that at some high enough drop velocity, the drop can simply jump over the groove with only minor suction.

The suction rate in the groove is influenced also by gravity. This effect is governed by the Bond number defined as

$$
\mathrm{Bo}=\frac{\varrho g \delta^{2}}{\sigma} .
$$

In Figure 13 a nomogram of the drop-groove interaction is shown for various Weber and Bond numbers. Only the cases of capture have been considered when the rear side of the groove has been wetted. The cases of immediate capture are not shown, since their operational window was already determined in Figure 12. The two lines in Figure 13 correspond to two thresholds, roughly estimated from the experimental data. One is the upper bound for drop capture and the second is the lower bound for drop passing. For higher Bond numbers the minimum threshold Weber number at which the drop still passes the groove, increases. For Weber numbers below the upper bound for capture, the probability for capture also increases with the Bond number.

The results in Figure 13 clearly demonstrate that the dimensionless parameters Bo and We are not sufficient to completely separate the phenomenon of drop capture after downstream wetting. This phenomenon is simply extremely sensitive to many additional influencing parameters which are not controlled in these experiments. Among these parameters could be the distribution of the roughness of the substrate and 


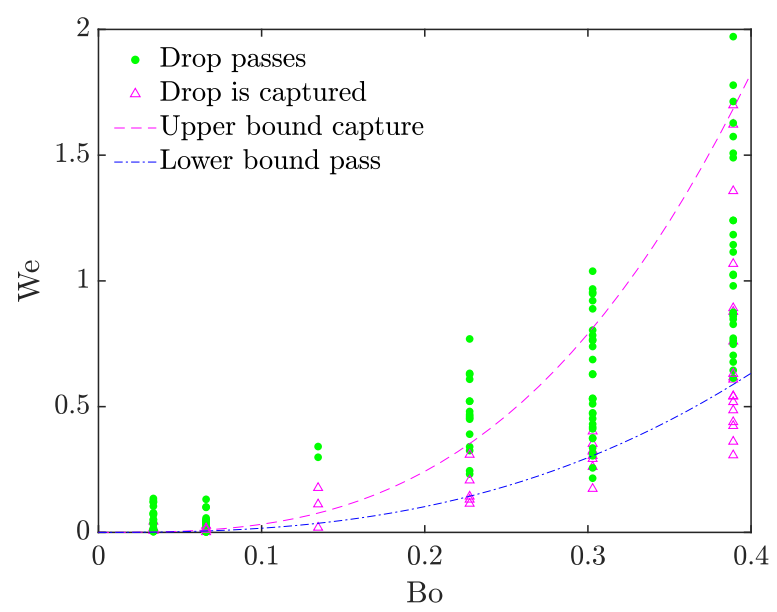

Fig. 13. A nomogram of the two major drop outcomes for different Weber and Bond numbers. The dashed line corresponds to the upper bound for drop capture and the dashdotted line to the lower bound for drop passing. Cases of immediate capture are not shown on this nomogram. Their appearance window is determined in Figure 12.

groove, the curvature at the corners, the three-dimensional distortion of the contact line as well as the 3D flows occurring at corner, for example cusp or rivulet formation $[19,20]$. The influence of these parameters can be counter-intuitive as can be seen in the examples shown in Figure 11. For larger, $15 \mu \mathrm{l}$ drop, the reduction of the groove width leads to the enhancement of the passing outcome. But smaller, $5 \mu \mathrm{l}$ drop, is captured at smaller groove widths. This surprising result can be explained only by the effect of the drop oscillations observed during its propagation, especially for smaller value of the capillary number. The drop is captured due to the downstream wetting if the groove width is comparable with the typical length of the drop fluctuations. These phenomena and these influencing factors have to be investigated in more detail in the future studies.

\section{Conclusion}

The interaction of an aerodynamically driven, wall-bound drop with a groove is governed by aerodynamic, capillary, viscous, gravitational and inertial forces. The present study identifies three major outcomes of the interaction. An appropriate scaling is proposed which can predict thresholds for determining which outcome will occur. One of these parameters is the ratio of the characteristic time for drop suction into the groove to the time of drop passing unobstructed over the groove. This parameter provides a condition for immediate drop capture into the groove.

However the conditions for drop passing over the groove are more complicated. The drop passes the groove for a certain combination of Weber and Bond numbers. Two thresholds are determined in this study. The upper bound corresponds to the lowest possible Weber number at which the drop definitely passes the groove. The lower bound corresponds to the highest Weber number at which drop is definitely captured.

In the intermediate region of the Weber and Bond numbers, between the upper and lower thresholds, both outcomes, passing and capturing of the drop, have been observed with some probability. For this range of the parameters, as well as for other 
groove geometries, a reliable prediction of the outcome can presently be performed only using numerical simulations [21].

Open access funding provided by Projekt DEAL. This work was financially supported by Adam Opel AG. The authors thank F. Werner and P. Klaus of Adam Opel AG, who provided feedback and valuable input for the experiments, and Andreas Mayrhofer for his assistance in the experiments. The authors are also kindly acknowledge the financial support by the German Research Foundation (DFG) within the Collaborative Research Centre 1194 "Interaction of Transport and Wetting Processes".

Open Access This is an open access article distributed under the terms of the Creative Commons Attribution License (http://creativecommons.org/licenses/by/4.0), which permits unrestricted use, distribution, and reproduction in any medium, provided the original work is properly cited.

Publisher's Note The EPJ Publishers remain neutral with regard to jurisdictional claims in published maps and institutional affiliations.

\section{References}

1. S. Tarquini, C. Antonini, A. Amirfazli, M. Marengo, J. Palacios, Cold Reg. Sci. Tech. 100, $50(2014)$

2. T. Theodorsen, W.C. Clay, Technical report 403, National Advisory Committee for Aeronautics (Langley Aeronautical Lab., Langley Field, VA, USA, 1933), document ID 19930091477

3. T. Hagemeier, M. Hartmann, D. Thévenin, Int. J. Multiph. Flow 37, 860 (2011)

4. C. Neinhuis, W. Barthlott, Ann. Bot. 79, 667 (1997)

5. A. Marmur, Langmuir 20, 3517 (2004)

6. K.J. Stout, L. Blunt, Int. J. Mach. Tool Manu. 41, 2039 (2001)

7. P. Dimitrakopoulos, J.J.L. Higdon, J. Fluid Mech. 336, 351 (1997)

8. A. Milne, A. Amirfazli, Langmuir 25, 14155 (2009)

9. S. Madani, A. Amirfazli, Colloids Surf. A Physicochem. Eng. Aspects 441, 796 (2014)

10. P.M. Seiler, M. Gloerfeld, I.V. Roisman, C. Tropea, Phys. Rev. Fluids 4, 024001 (2019)

11. M. Dianat, M. Skarysz, A. Garmory, Int. J. Multiph. Flow 91, 19 (2017)

12. M. Dianat, M. Skarysz, G. Hodgson, A. Garmory, M. Passmore, SAE Int. J. Passeng. Cars - Mech. Syst. 10, 369 (2017)

13. P.M. Seiler, Aerodynamically driven wall-bounded drop motion and rivulet formation, Ph.D. thesis, Technische Universität, 2019

14. A. Trujillo-Pino, K. Krissian, M. Alemán-Flores, D. Santana-Cedrés, Image Vis. Comput. 31, 72 (2013)

15. S.F. Chini, A. Amirfazli, Colloids Surf. A 388, 29 (2011)

16. M.J. Shelley, F.R. Tian, K. Wlodarski, Nonlinearity 10, 1471 (1997)

17. J. Bohr, S. Brunak, T. Nørretranders, Europhys. Lett. 25, 245 (1994)

18. M.B. Amar, D. Bonn, Physica D 209, 1 (2005)

19. V.T. Gurumurthy, D. Rettenmaier, I.V. Roisman, C. Tropea, S. Garoff, Colloids Surf. A 544, $118(2018)$

20. F. Gerlach, M. Hartmann, C. Tropea, Colloids Surf. A 583, 123977 (2019)

21. D. Rettenmaier, Numerical simulation of shear driven wetting, Ph.D. thesis, Technische Universität Darmstadt, Darmstadt, Germany, 2019 